\title{
Early stage and long term treatment of multiple sclerosis with interferon- $\beta$
}

\author{
This article was published in the following Dove Press journal: \\ Biologics:Targets \& Therapy \\ 29 May 2009 \\ Number of times this article has been viewed
}

\author{
Angela Applebee \\ Hillel Panitch \\ Department of Neurology, University \\ of Vermont College of Medicine, \\ and Neurology Service, Fletcher Allen \\ Health Care, Burlington, VT, USA
}

\begin{abstract}
Multiple sclerosis (MS) affects young adults during the most productive years of their lives, and until recently many neurologists were limited to treating symptoms and attacks without any ability to alter the disease course. The 1990s ushered in an era of possibility with the approval of three interferon-beta (IFN $\beta$ ) therapies for the treatment of MS. Though the mechanism of action of these agents is not completely understood, it is clear they reduce magnetic resonance imaging (MRI) activity as well as improve clinical outcomes. The principal randomized, blinded, multicenter trials of IFN $\beta$ all point to the need for early treatment soon after the diagnosis of MS is made. Efficacy has also been shown in patients treated after a first demyelinating event. Data on IFN $\beta$ in the treatment of secondary progressive MS (SPMS) is not impressive, although it shows some benefit in SPMS patients who continue to experience MRI activity and clinical relapses, signifying a continued inflammatory component to their disease. There has been no proven efficacy of IFN $\beta$ in the treatment of primary progressive MS (PPMS). The IFN $\beta$ therapies are generally well tolerated with a favorable side effect profile. Despite benefits in MRI and clinical measures such as relapse rates and Expanded Disability Status Scale progression, patients continue to exhibit clinical progression and radiological atrophy, pointing to confounding factors and perhaps multiple etiologies of a disease that is not yet fully understood. In addition, the subject of neutralizing antibodies has recently assumed importance. The significance of these on treatment efficacy is uncertain, and until a universally accepted reliable assay is adopted, the decision to change treatment continues to rely on the clinical interpretation of the patient's history and physical examination. Additional recommendations for management of patients, informed by the best available evidence, are also presented.
\end{abstract}

Keywords: interferon- $\beta$, multiple sclerosis, clinical trials, neutralizing antibodies

\section{Introduction}

Multiple sclerosis (MS) is characterized by unpredictable signs and symptoms involving the sensory, motor, visual, and brainstem systems. Recently, cognitive and mood disturbances have also become more widely recognized in these patients. The disease is also highly variable in terms of the frequency and severity of attacks, as well as the extent and rate of progression of disability. Despite this unpredictability, neurologists categorize patients into one of four forms based on disease course. The currently accepted classification of MS into four forms was established by an international survey. ${ }^{1}$ The most common form is relapsing remitting MS (RRMS), which accounts for $85 \%$ of MS cases at onset. This is characterized by relapses, also known as attacks or exacerbations, followed either by full recovery, or a residual neurological deficit. Relapses are defined as neurologic symptoms lasting more than 24 hours, preceded by at least one month
Correspondence: Hillel Panitch Neurology Service, UHC Campus, I South Prospect Street, Burlington, VT 0540I, USA

Tel + I 802847544 I

Fax + I 80284725 I 2

Email hillel.panitch@vtmednet.org 
of stability, and accompanied by a change in the neurologic examination that cannot be explained by an infection or other illness. The time between attacks is characterized as a remission or lack of disease progression whether or not the patient's neurologic status returns to baseline. Approximately 50\% of RRMS patients will go on to develop secondary progressive MS (SPMS) within 10 years. SPMS is defined by an initial relapsing remitting course, followed by gradual progression with or without occasional relapses or plateaus. These patients are usually more disabled than RRMS patients, and the disability results from incomplete recovery from exacerbations as well as steady progression.

Patients with primary progressive MS (PPMS) make up approximately $10 \%$ of the population. PPMS patients have disease progression from onset, sometimes with infrequent plateaus or minor improvements. The hallmark of PPMS is continued worsening of the baseline without clear relapses. Progressive relapsing MS (PRMS) is the rarest form, compromising approximately $5 \%$ of MS patients. These individuals have a progressive course from disease onset with one or more superimposed acute relapses, following which the progression continues. A related condition is the clinically isolated syndrome (CIS), defined as a single demyelinating event suggestive of an initial MS attack. These patients are at risk of developing RRMS, but do not meet currently accepted criteria for MS because of a lack of clinical or radiologic episodes separated in time and space. Patients with T2 magnetic resonance imaging (MRI) lesions at onset have a substantially greater risk of developing MS and progression of disability, than those with normal MRI scans. ${ }^{2}$

Treatment of MS patients can be divided into three types: acute, disease-modifying, and symptomatic. Prior to the early 1990s, management of MS patients was limited to acute and symptomatic treatment, leaving neurologists at a loss as to what to do between attacks. In 1993, the first diseasemodifying agent for the treatment of MS was approved by the US Food and Drug Administration (FDA), and over the course of the next few years, two other IFN $\beta$ agents gained FDA approval for treatment of relapsing forms of MS. There is still much to be learned regarding the pathogenesis of this disease, one clear aspect being that a component of immune dysregulation occurs which responds moderately well to immunomodulating treatment. A complete review of all the disease-modifying therapies, or even all aspects of IFN $\beta$, is beyond the scope of this review. Our aim, rather, is to address briefly the proposed mechanisms of action of the IFN $\beta$ preparations, describe their proven efficacy and safety in clinical trials, discuss the importance of neutralizing antibodies and other safety issues, and conclude with therapeutic recommendations based on these factors, and on our own experience, for the treatment of patients with MS.

\section{Mechanism of action}

Experimental evidence supports the hypothesis that the MS disease process is due, at least in part, to autoimmunity. ${ }^{3-5}$ The currently prevalent theory involves the activation of autoreactive type $1 \mathrm{~T}$ helper (Th1) cells in the peripheral immune system. Th1 cells are exposed either to self antigens or to molecules that closely mimic them, and become activated in the presence of co-stimulatory molecules, leading to the release of proinflammatory cytokines such as interleukin 12 (IL-12), IL-17, interferon gamma (IFN $\gamma$ ), and tumor necrosis factors. Associated with this, there is an up-regulation of adhesion molecules allowing for binding of $\mathrm{T}$ cells and transmigration across the endothelial surface of CNS venules. The activated $\mathrm{T}$ cells then attack the basement membrane component of the blood-brain barrier (BBB) by secreting matrix metalloproteinases (MMP), proteolytic enzymes that increase $\mathrm{BBB}$ permeability and allow for passage of the $\mathrm{T}$ cell into the CNS. This process is then amplified upon entry into the CNS by presentation of myelin autoantigens and further production of inflammatory cytokines, leading to the destruction of myelin and axons. This is, of course, a greatly simplified view of the process, many aspects of which are still under investigation.

There are multiple mechanisms by which IFN $\beta$ is proposed to work, beginning with down regulation of major histocompatibility complex type 2 (MHC II) expression. The antigen-MHC II complex is recognized on the surface of an antigen presenting cell by a Th1 cell, leading to activation. Downregulation of MHC II leads to decreased presentation of self antigens and decreased Th1 activation. IFN $\gamma$, a pro-inflammatory cytokine, usually up-regulates MHC II complexes and IFN $\beta$ is thought to interfere with this process. ${ }^{6}$ IFN $\beta$ also affects IFN $\gamma$ another way, by decreasing its release from activated T cells. ${ }^{7}$ IFN $\beta$ has been shown to decrease the co-stimulatory molecules necessary to activate T cells, ${ }^{8}$ and may also bring about a shift from a pro-inflammatory (Th1) state to an anti-inflammatory state characterized by proliferation of type 2 (Th2) T helper cells. A recent study showed a shift of cytokines from those that promote inflammation, eg, IL-12, to anti-inflammatory cytokines, such as IL-10, thus supporting a shift from a Th1 to a Th2 type milieu. ${ }^{9}$

IFN $\beta$ can affect BBB permeability to cells and proteins via multiple mechanisms, the most important of which is probably inhibition of MMP production by $\mathrm{T}$ cells, thereby 
preventing breakdown of the subendothelial extracellular matrix and passage of activated $\mathrm{T}$ cells into the CNS. ${ }^{10,11}$ This effect is most vividly demonstrated by the dramatic reduction in gadolinium-enhancing lesions seen on MRI almost immediately after treatment with IFN $\beta$ is begun. ${ }^{12}$ Interferons were also recently found to demonstrate a direct stabilizing effect on endothelial cells in vitro limiting the migration of small particles into the CNS. ${ }^{13}$ Another potential mechanism involves the adhesion of activated $\mathrm{T}$ cells to the endothelial surface. Patients receiving IFN $\beta$ have been shown to produce soluble vascular cell adhesion molecule (VCAM), corresponding to a reduction in contrast enhancing lesions on MRI. ${ }^{14,15}$ Thus soluble VCAM may act as a decoy, binding to activated $\mathrm{T}$ cells and keeping them from crossing into the CNS.

IFN $\beta$ also inhibits the expansion of $\mathrm{T}$ cell clones ${ }^{16}$ and was found to increase $\mathrm{T}$ cell death through inhibition of FLIP, an antiapoptotic protein. ${ }^{17}$ Other investigators have focused on regulation of cytokine and growth factor production in MS. For example, IFN $\beta$ may promote production of the suppressive cytokine IL-10 by activated T cells in early MS, but not in later stage progressive forms of the disease. ${ }^{18}$ Effects on IFN $\gamma$ and brain-derived neurotrophic factor (BDNF) have also been studied, with interesting but sometimes paradoxical results that require further analysis. ${ }^{19}$

Researchers have also turned their attention to molecular mechanisms by which interferon therapy may exert its action. Because interferons act by promoting gene activation via intracellular transcription factors, changes in mRNA expression can be identified in patients exposed to interferon therapy. Many studies, eg, ${ }^{20}$ have focused on expression of genes thought to be involved in MS. Recently, however, investigators have attempted to cast a broader net, using cDNA microarrays to detect changes in gene expression related to IFN $\beta$ therapy, ${ }^{21}$ or to identify responders and nonresponders to IFN $\beta$ therapy. ${ }^{22}$ Although this area of research is preliminary, it may represent an important step toward linking MS etiology and therapy. ${ }^{23}$

Currently, there are two forms of IFN $\beta$ approved for the treatment of MS, both of which are produced by recombinant DNA technology. IFN $\beta$-1a (Avonex ${ }^{\circledR}$, Biogen Idec and Rebif $^{\circledR}$, Merck Serono) is produced in Chinese hamster ovary cell lines. These molecules are glycoproteins with a 166 amino acid sequence, identical to that of human IFN $\beta$; however, the glycosylation patterns are different, and not necessarily identical to those found in human IFN- $\beta$. IFN $\beta-1 b$ (Betaseron $^{\circledR}$, Bayer HealthCare or Betaferon ${ }^{\circledR}$, Schering AG) is produced in an Escherichia Coli cell line and is not glycosylated, as bacteria cannot glycosylate proteins. IFN $\beta-1 b$ has an amino acid substitution, serine for cysteine at position 17 , to prevent aggregation and preserve proper folding and biologic function. This molecule also differs slightly from IFN $\beta$-1a in length, as it contains only 165 amino acids due to deletion of the N-terminal methionine. The differences between IFN $\beta-1 b$ and IFN $\beta$-1a have certain consequences that are thought to be important, such as lower specific activity of IFN $\beta-1 b$, as well as an increased tendency to neutralizing antibody (NAb) formation in patients treated with IFN $\beta-1 b$. The 1990s saw several randomized controlled trials addressing the efficacy of IFN $\beta$ in the treatment of multiple sclerosis. Tables 1 and 2 list the large multicenter studies for the treatment of MS and CIS with the different forms of IFN $\beta .^{24}$

\section{Efficacy of IFN $\beta$ in MS}

The first pilot study of IFN $\beta$ - $1 \mathrm{~b}$ for the treatment of MS was performed in the late $1980 \mathrm{~s}^{25}$ Thirty RRMS patients were randomized to receive $0.8,4,8$, or 16 million international units (MIU) of IFN $\beta-1$ b or placebo via subcutaneous (SC) injection administered three times per week (TIW). The 8 MIU dose was reasonably well tolerated; however, the 16 MIU caused excessive side effects. This study showed a dose dependent reduction in attacks, but an effect of treatment on disease progression could not be assessed.

A phase III trial of IFN $\beta-1 b$ in MS was initiated in $1988 .{ }^{26}$ The primary outcome measures were reduction in annual exacerbation rate and proportion of exacerbation free patients. Three hundred seventy-two clinically definite RRMS patients were enrolled at 11 centers in the United States and Canada. Patients were between the ages of 18 and 50, had a history of two exacerbations within the previous two years, and an Expanded Disability Status Scale (EDSS) score ${ }^{27}$ of 5.5 or less. Subjects were randomized to receive placebo, high dose ( $8 \mathrm{MIU})$, or low dose (1.6 MIU) of IFN $\beta$-1b by SC injection every other day. MRI scans were performed yearly with the exception of a subset of patients at a single site who were scanned every six weeks for the first two years to assess the effect of treatment more closely. This study was initially planned for two years, was subsequently extended to three years, and then to five years. The three-year data revealed statistically significant results in the treatment arms compared to placebo. ${ }^{26,28}$ The annual exacerbation rate for the high dose group (0.84) was less than for the placebo group (1.27), with a $\mathrm{p}$ value of 0.0001 , approximately a one-third reduction in the frequency of exacerbations. Moderate and severe attacks were decreased by $50 \%$ compared to placebo. 


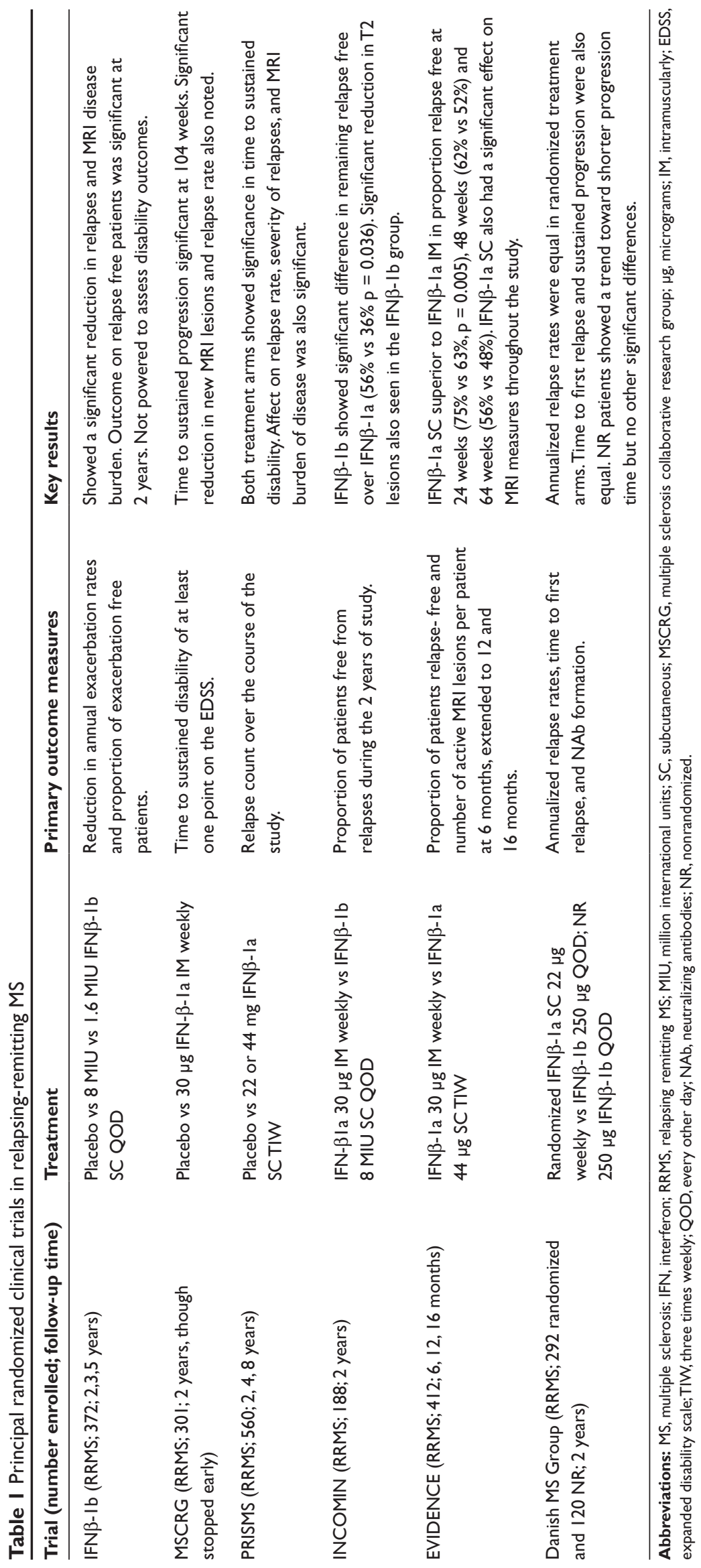


The number of exacerbation-free patients at two years was statistically significant in the high dose group; however this was not maintained at three years. The effect of IFN $\beta$ - 1 b on disease progression, as measured by change in EDSS scores, did not reach statistical significance. Inclusion of the low dose group revealed a dose-response effect, with values for most outcome measures falling between the placebo and high-dose groups.

Cumulative disease burden (an approximation of lesion volume) on MRI showed a highly significant effect of high dose IFN $\beta-1 b$ compared to placebo $(p=0.002)$. MRI activity assessed by measuring new or enlarging lesions in the subset of patients undergoing frequent MRI scanning every six weeks for two years showed an $80 \%$ reduction of activity with high dose treatment. In a subsequent report ${ }^{29}$ the median time on study for patients was 4 years, with a few reaching 5 years. When all data were pooled for all time points in the study, a $30 \%$ reduction in relapse rate persisted in the high dose treatment arm $(0.78$ per year compared with 1.12 for placebo; $p=0.0006)$. Although the exacerbation rates each year were approximately one-third lower in the high dose treatment arm, after year 2 this was not statistically significant, probably due to dropouts and loss of statistical power. The MRI efficacy of IFN $\beta-1 b$ persisted through year 5, with no significant increase in lesion burden. The study was not powered to show a significant effect on disease progression. Both doses of IFN $\beta$ - $1 \mathrm{~b}$ were tolerated well enough for the FDA to grant approval without undue concern for serious adverse events.

IFN $\beta-1 b$ was subsequently tested in patients with SPMS with conflicting results in two placebo-controlled multicenter randomized studies. In the European study, ${ }^{30} 718$ patients with SPMS and EDSS scores of 3.0 to 6.5, with confirmed progression or at least two exacerbations in the prior two years, were randomized to either $8 \mathrm{MIU}$ of IFN $\beta-1 \mathrm{~b}$ or placebo every other day. The primary outcome was time to confirmed progression of disability, as measured by a one-point increase on the EDSS, sustained for three months (or a 0.5-point increase if the baseline EDSS was 6.0 or 6.5). The study was terminated shortly before its planned end point of three years because of statistically convincing evidence of efficacy. A significant difference in time to confirmed progression was found in favor of IFN $\beta-1 b(p=0.0008)$. The IFN group had a $21.7 \%$ reduction in rate of progression, or a 12-month delay in reaching similar disability levels compared with placebo. This effect was also seen in time to becoming wheelchair bound (EDSS 7.0 or greater) being delayed by nine months. There were significant reductions in relapse rates and in MRI activity as measured by contrast enhancing lesions; however, there was no reduction in brain atrophy. The results of this study led to the approval of IFN $\beta-1 b$ in Europe and Canada for the treatment of patients with SPMS.

The second trial of IFN $\beta-1 b$ in SPMS was performed in the United States and Canada. ${ }^{31}$ Patients eligible for randomization had EDSS scores of 3.0-6.5, and an increase in their EDSS scores of at least one point in the two years prior to screening. There was no relapse requirement other than a history of one relapse at some time in the past, to confirm the diagnosis of SPMS. Nine hundred thirty-nine patients were randomized to IFN $\beta-1 \mathrm{~b} 8 \mathrm{MIU}$, or a variable dose of $5 \mathrm{MIU} / \mathrm{m}^{2}$, or to one of two matching placebo arms administered SC every other day. The planned outcome measures were the same as in the European study. This trial was planned for a three year end-point, but was stopped early because an interim data analysis indicated the inability to achieve a significant difference in the primary outcome measure. In contrast to the European study, treatment with IFN $\beta$-1b did not improve disability measures compared with placebo. Consistent with previous studies, there was a significant reduction in the already low relapse rates and in MRI measures of activity.

The disparity between these two studies has been the subject of much debate. When differences in baseline demographics were analyzed, ${ }^{32}$ patients in the European trial were found to be younger, to have higher relapse rates prior to entry, shorter disease duration, and a higher proportion of contrast enhancing lesions. The entry criteria for these two studies may have added to the differences seen, as the European trial required either two relapses or a one-point increase in the EDSS over two years to document disease progression, whereas the North American trial excluded patients with relapses and required a one-point deterioration in two years. In the European study, this may have selected for patients earlier in the disease course, characterized by more intense inflammation, and therefore a greater tendency to respond to IFN $\beta-1$ b. Kappos and colleagues (2004) also remarked on the lack of approved therapies in Europe for the treatment of MS at the time of enrollment in the trial. In North America, during enrollment of the SPMS trial there was already one approved immunomodulatory therapy, and two others became available during the course of the study. Thus, patients in the North American group may have been preselected for a lower likelihood of responding to IFN $\beta-1 b$. The authors of the retrospective analysis concluded that SPMS patients with continuing superimposed relapses may be more likely to benefit from IFN $\beta-1 b$ treatment. 


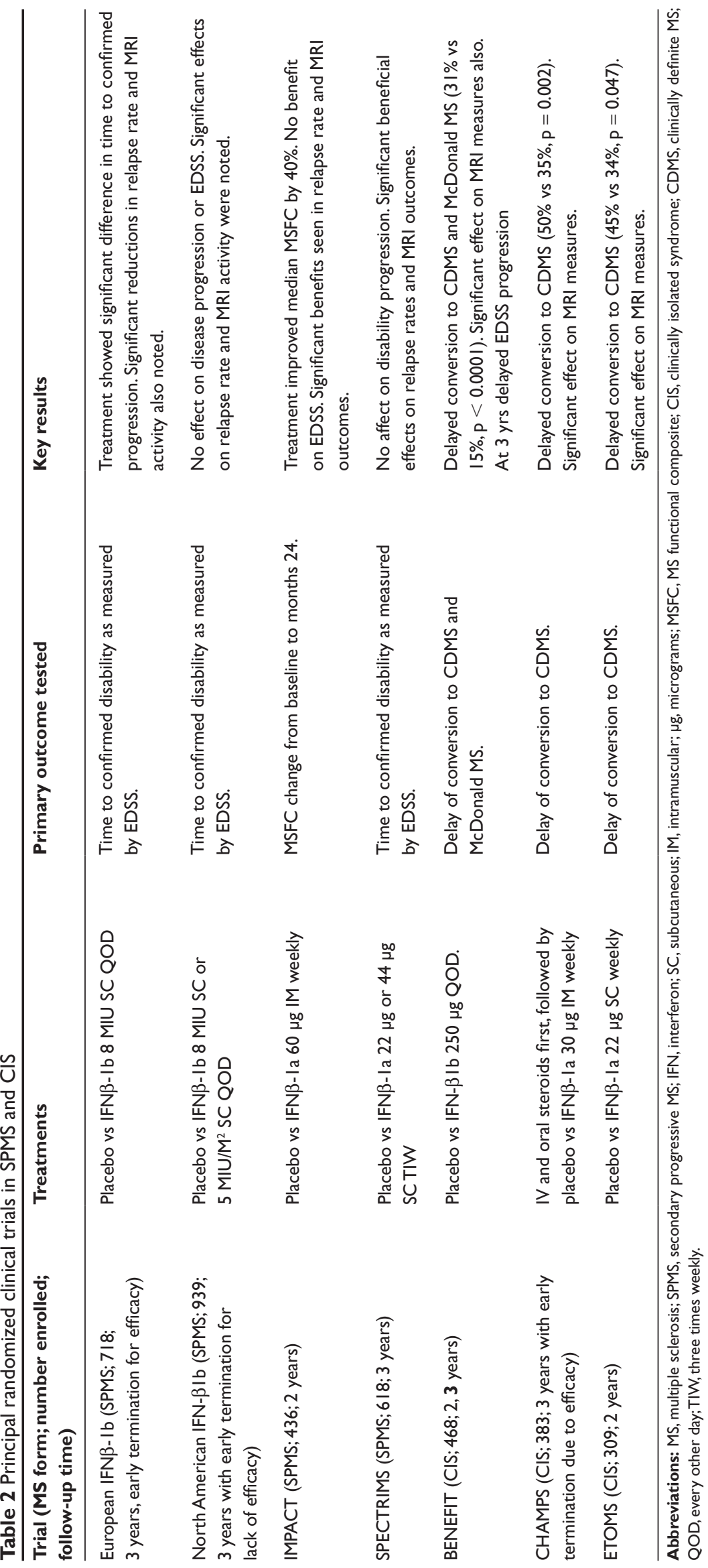


The efficacy and tolerability of IFN $\beta$-1b in the treatment of clinically isolated syndromes (CIS) was recently reported..$^{33}$ The Betaferon in Newly Emerging Multiple Sclerosis for Initial Treatment (BENEFIT) trial was a multicenter, randomized, double-blind, placebo controlled trial assessing the effect of treatment on the rate of conversion to clinically definite MS (CDMS) as defined by the Poser criteria ${ }^{34}$ and furthermore, as defined by the newer McDonald criteria. ${ }^{35}$ Four hundred sixty-eight patients with a first clinical demyelinating event (either monosymptomatic or polysymptomatic) and at least two clinically silent MRI lesions were randomized in a five to three ratio to receive either $8 \mathrm{MIU}$ of IFN $\beta-1 \mathrm{~b}$ or placebo subcutaneously every other day. Exacerbations were documented by an examining physician and when the diagnosis of CDMS was confirmed, the patient was offered open-label treatment. IFN $\beta$-1b significantly delayed the onset of CDMS and "McDonald MS" ( $p<0.0001)$. At the end of the two year study, the probability of not developing "McDonald MS" was twice as high with IFN $\beta-1 b$ (31\%) as with placebo (15\%). IFN $\beta$-1b significantly reduced MRI activity and was well tolerated, as indicated by the low dropout rate $(7.2 \%)$ in the IFN $\beta-1 b$ group.

Following the two year placebo-controlled phase, patients were eligible to enter an open-label follow-up phase and were offered IFN $\beta-1 \mathrm{~b} 250 \mu \mathrm{g}$ subcutaneously every other day for up to five years from randomization. ${ }^{36}$ Follow-up visits occurred every six months with a blinded examiner performing a standardized neurological assessment and EDSS score. The primary outcomes of this analysis were time to diagnosis of CDMS, time to confirmed EDSS progression, and score on a patient-reported functional assessment scale. Eighty-nine percent of patients entered the follow-up phase with $84 \%$ completing three years. At that time, early treatment was shown to reduce the risk of developing CDMS by $41 \%$ (absolute risk reduction of $14 \%$ ) compared to delayed treatment. More importantly, early treatment reduced the risk for progression of disability, as measured by a sustained one point change in the EDSS, by $40 \%$ compared to the delayed treatment group.

The pivotal trial of IFN $\beta-1$ a $\left(\right.$ Avonex $\left.^{\circledR}\right)$ was the first to use delay of disability, as measured by time to progression of the EDSS score by one point sustained for six months, as the primary outcome measure. ${ }^{37}$ The investigators enrolled 301 relapsing MS patients with at least two exacerbations during the previous three years, and EDSS scores of 1.0 to 3.5 . Patients were randomized to receive either IFN $\beta$-1a 6 MIU $(30 \mu \mathrm{g})$ or placebo by weekly intramuscular (IM) injection. This study was designed with a two-year end-point in mind; however, the number of dropouts was lower than anticipated, and the study was terminated early. Only $57 \%$ of patients completed two years, and $77 \%$ completed 18 months. Time to sustained progression was significantly greater in IFN $\beta-1$ a treated patients $(21.9 \%)$ compared with placebo $(34.9 \%)$ at 104 weeks $(p=0.02)$. There was a significant reduction in relapse rates, most evident at the end of year 2 . This reduction was $31 \%$ for those completing two years, but only $18 \%$ for all patients in the study.

A significant reduction in gadolinium enhancing lesions was present at year 1 , and was maintained at year $2(p=0.02)$, but the effect on total T2 lesion volume was not significant. Tolerance of the drug was acceptable, considering the inherent discomfort of the IM injection route. Subsequent re-analysis of the data by the investigators ${ }^{38}$ included a change of EDSS greater than two points, or sustained progression by one point for one year, with the conclusion that the previous results underestimated the effect of IFN $\beta-1$ a. Further post-hoc analysis revealed benefit in some areas of neuropsychological testing and degree of brain atrophy in patients treated with IFN $\beta-1 \mathrm{a} .{ }^{39,40}$

Although IM IFN $\beta$-1a was modestly effective in the treatment of relapsing MS patients, the optimal dose was not established. A randomized, double-blind, dose-comparison study of weekly IFN $\beta$-1a comparing $30 \mu \mathrm{g}$ versus $60 \mu \mathrm{g}$ by weekly IM injection was therefore conducted. ${ }^{41}$ The primary endpoint was six-month EDSS progression, and there were multiple other clinical and MRI secondary outcome measures. Eight hundred two relapsing MS patients with EDSS scores of 2.0 to 5.5 were enrolled and randomized to either treatment arm. At 36 months, there was no statistical difference between the two treatment arms in either primary or secondary endpoints. 608 patients completed 36 months, and 448 patients remained in an extension of the trial to 48 months. At that time, there continued to be no statistical difference between the $30 \mu \mathrm{g}$ and $60 \mu \mathrm{g}$ weekly doses in terms of efficacy or safety. ${ }^{42}$ In the absence of a placebo group, the meaning of these findings is dubious.

The effect of IM IFN $\beta$-1a in SPMS was evaluated in the International Multiple Sclerosis Secondary Progressive Avonex Clinical Trial (IMPACT), which enrolled 436 SPMS patients with progression over the previous year, and EDSS scores of 3.5 to $6.5 . .^{43}$ This trial was designed to determine whether $60 \mu \mathrm{g}$ IFN $\beta$-1a given once weekly by the IM route was more effective than placebo as measured by changes at two years in the MS Functional Composite (MSFC), a novel measure of disability. ${ }^{44}$ IFN $\beta$-1a treatment reduced median MSFC worsening by $40 \%(\mathrm{p}=0.033)$. This effect 
was primarily driven by the nine-hole peg test and the paced auditory serial addition test. No benefit was seen on the EDSS, a secondary endpoint. Significant benefits in relapse rate and MRI outcomes were noted in IFN-treated subjects. This was the first study to use the MSFC to assess disability, but because it had not yet been validated as an outcome measure, the FDA refused to approve IM IFN $\beta$-1a for the treatment of SPMS.

The Controlled High-Risk Avonex Multiple Sclerosis Prevention Study (CHAMPS) trial ${ }^{45}$ sought to determine whether patients with CIS treated with $30 \mu \mathrm{g}$ of IFN $\beta-1 \mathrm{a}$ intramuscularly once weekly are less likely to develop CDMS. This randomized double-blind study enrolled 383 patients with a first isolated demyelinating event of optic neuritis, incomplete transverse myelitis, or brainstem - cerebellar dysfunction. Patients were also required to have two or more T2 signal abnormalities consistent with demyelination on their MRI scans. Patients were screened within two weeks of symptom onset and all received a three-day course of one gram of intravenous methylprednisolone followed by 14 days of oral prednisone. Treatment with IFN $\beta$-1a began within four weeks of the initial event. The primary outcome measure was conversion to CDMS, which required documentation of new symptoms suggesting a relapse, and confirmation by a central end-point committee. MRI scans were performed every six months for the first 18 months, but were not required to confirm the diagnosis of CDMS. When CDMS was confirmed, subjects were removed from the study and offered treatment with IFN $\beta-1$ a. This trial was terminated early because of significant evidence of efficacy in the treatment group compared to controls. There was a cumulative probability of $50 \%$ of placebo patients having a second attack, compared to $35 \%$ of those treated with IFN $\beta-1 \mathrm{a}(\mathrm{p}=0.002)$. Changes in the volume of brain lesions on T2 weighted MRI were statistically significant in the treatment arm as well. Following publication of this study, the FDA approved the use of IM IFN $\beta$-1a for the treatment of CIS.

In a small randomized controlled trial of PPMS patients treated with IFN $\beta-1 \mathrm{a},{ }^{46}$ fifty patients were randomized to weekly IM injections of IFN $\beta$-1a at doses of $30 \mu \mathrm{g}, 60 \mu \mathrm{g}$, or placebo for two years. The primary endpoint of sustained progression in disability was not met in either active treatment arm. Larger studies in PPMS have not been performed.

The pivotal study of IFN $\beta$-1a (Rebif $®)$ given by SC injection was PRISMS (Prevention of Relapses and Disability by Interferon $\beta$-1a Subcutaneously in Multiple Sclerosis), a multicenter, randomized, placebo controlled trial of 560 patients with RRMS. ${ }^{47}$ Patients were eligible if they had had at least two relapses in the preceding two years and EDSS scores between 0 and 5.0. They were randomized to either $22 \mu \mathrm{g}, 44 \mu \mathrm{g}$, or placebo treatment arms, and were treated TIW for two years. The primary outcome measure was relapse count over the course of the study. Both treatment groups had significant reductions in relapse rate compared with controls, achieving $27 \%$ and $33 \%$ reductions for the $22 \mu \mathrm{g}$ and $44 \mu \mathrm{g}$ doses respectively over the study period ( $p<0.0002)$. Both doses also showed significance in percentage of relapse free patients, time to first relapse, and severity of relapses. Time to sustained progression was significantly longer $(\mathrm{p}<0.05)$ in both interferon IFN $\beta$-1a treatment groups than in the placebo group. A composite score of integrated disability (area under the EDSS curve over time) showed a $77 \%$ reduction in accumulated burden of disability during the study. The number of enhancing lesions as well as T2 lesion volume on MRI were significantly lower in both treatment groups $(\mathrm{p}<0.0001) .{ }^{48}$ Although the higher dose group did better, there was no statistical difference between the two doses on major clinical outcomes.

After completion of the initial two-year PRISMS trial, the study was extended (PRISMS-4) and patients initially receiving placebo were re-randomized to either high or low dose IFN $\beta-1 a .{ }^{49}$ Those already on interferon therapy remained on their current dose. The primary outcome was relapse count per patient over four years. Ninety percent of patients randomized in the initial trial were enrolled in PRISMS-4. At the end of four years, there was continued evidence of efficacy, and a dose effect became more apparent, particularly on MRI outcomes. Most notably, confirmed progression of disability was lowest in the patients who took high dose IFN $\beta$-1a for four years, and highest in those who received placebo for two years followed by low dose IFN $\beta$-1a for the next two years. The effect was intermediate for the other two groups, supporting the idea of starting therapy as soon as possible after diagnosis.

A long-term follow up (LTFU) at 7-8 years of the PRISMS study was recently published. ${ }^{50}$ Sixty-eight percent (382/560) of patients from the original PRISMS trial returned for assessment. At LTFU, 80\% of the cohort had not progressed to SPMS, and the mean T2 lesion load and relapse rate remained lowest in those treated with high dose IFN $\beta$-1a for eight years. Interestingly, brain parenchymal volume, as a measure of atrophy, declined in all groups. This measurement was limited by the lack of a placebo cohort for the full duration of the study. The authors commented that brain parenchymal volume was a promising new measure 
of chronicity of disease, but that it could be confounded by physiologic fluctuations. Importantly, there were no new safety concerns identified and long-term safety and tolerability were maintained. The inherent weaknesses in LTFU studies should be considered when interpreting these results.

The Once Weekly Interferon for MS Study (OWIMS) ran nearly concurrently with the PRISMS study. ${ }^{51}$ This was a randomized, double-blind study of IFN $\beta-1$ a $22 \mu \mathrm{g}, 44 \mu \mathrm{g}$, or placebo administered by SC injection weekly for one year. A total of 293 RRMS patients were enrolled. The primary outcome measure was the number of combined unique (CU) active lesions (PD/T2 or T1-enhancing) at 24 weeks. No clinical variables, other than steroid use in the $44 \mu \mathrm{g}$ group, reached statistical significance. The MRI features of CU lesions and burden of disease significantly favored the treatment arms. The authors concluded that when taken together with other studies, a dose-effect relationship exists for both clinical and MRI variables.

In 2001, a trial of SC IFN $\beta$-1a in SPMS was reported. ${ }^{52}$ This study enrolled 618 patients in Europe, Canada, and Australia with EDSS scores of 3.0 to 6.5, a history of clinical progression for at least six months, and an increase in EDSS of one or more points in the two previous years $(0.5$ if the EDSS was 6.0-6.5). Subjects were randomized to receive $22 \mu \mathrm{g}, 44 \mu \mathrm{g}$, or placebo by SC injection TIW for three years. The study's primary endpoint was time to confirmed disability progression, defined as an increase from baseline by at least 1 EDSS point (0.5 if baseline greater than 5.5) confirmed three months later. The primary outcome measure was not statistically affected by treatment, as was also seen in the North American IFN $\beta-1 b$ trial. There was a significant effect of treatment on relapse rates, and MRI measures including lesion load, and new or enlarging T2 lesions. ${ }^{53}$ A subgroup analysis suggested that maximal benefit with IFN $\beta$-1a treatment in SPMS was seen in patients who continued to experience relapses, tending to confirm the outcomes of the European and North American IFN $\beta-1 b$ studies.

A trial of IFN $\beta$-1a in patients with CIS was reported by the Early Treatment of Multiple Sclerosis (ETOMS) Study Group. ${ }^{54}$ Eligible patients experienced their first event suggestive of monosymptomatic or polysymptomatic demyelinating disease within 3 months, had one or more abnormalities present on neurologic examination, and an MRI consistent with demyelinating disease. Steroid treatment of the initial attack was allowed only for moderate or severe exacerbations. Three hundred eight patients were randomized to either placebo or $22 \mu \mathrm{g}$ IFN $\beta$-1a by
SC injection once weekly. The primary outcome measure was conversion to CDMS as defined by a second clinical relapse, confirmed when the evaluating physician could detect an objective change on neurologic examination. At the end of two years, $45 \%$ of placebo treated patients converted to CDMS, compared with $34 \%$ of those receiving active treatment $(\mathrm{p}=0.047)$. The treatment arm showed a significant effect on the number of T2 lesions and total lesion volume. Subsequently, in a reanalysis of the data, Eilippi and colleagues ${ }^{55}$ reported a significant treatment effect on brain atrophy at two years compared to baseline $(p=0.0031)$. This study may be interpreted as showing that when MS is treated in its earliest stages, even a dose of IFN $\beta$-1a that is considered subtherapeutic ( $22 \mu \mathrm{g}$ once weekly) may be effective in preventing or delaying relapses.

\section{Head-to-head studies}

There have been three head-to-head trials of IFN $\beta$ therapy published to date. The longest of these was the INCOMIN (Independent Comparison of Interferons) trial, comparing every other day SC IFN $\beta-1 b$ to once weekly IM IFN $\beta-1 a .{ }^{56}$ Patients included in this study had clinically definite RRMS, EDSS scores between 1.0 and 3.5, and two documented relapses within the previous two years. One-hundred eighty-eight patients enrolled at 15 Italian centers were randomized to either IFN $\beta$-1a $30 \mu \mathrm{g}$ IM weekly or IFN $\beta-1 \mathrm{~b} 8$ MIU SC every other day. The MRI evaluators were blinded, but clinical evaluators were not. The primary outcome measure was the proportion of relapse-free patients during the two years of study. The IFN $\beta$ - 1 b cohort showed a significant difference in freedom from relapses compared with IFN $\beta$-1a ( $56 \%$ vs $36 \% \mathrm{p}=0.036)$, and a significant reduction in new T2 lesions on blinded MRI ( $p<0.0003$ ).

In the EVIDENCE (Evidence of Interferon Dose-response: European North American Comparative Efficacy) trial, ${ }^{57}$ investigators compared the efficacy and safety of once weekly IFN $\beta$-1a $30 \mu \mathrm{g}$ IM with high-dose IFN $\beta$-1a $44 \mu \mathrm{g}$ SC TIW. The 677 patients enrolled in this study had definite RRMS, EDSS scores of 0 to 5.5, were IFN naïve, and had experienced two or more exacerbations within the previous two years. The patients were aware of their treatment assignment, but evaluators of the clinical and MRI outcomes were blinded. The primary clinical endpoint was the proportion of relapsefree patients, and the primary MRI endpoint was the number of active lesions per patient per scan at six months, later extended to 12 and 16 months. ${ }^{58}$ Patients were evaluated every four weeks during the initial 24-week period, and again at 36,48 , and 64 weeks. The $44 \mu \mathrm{g}$ treatment group showed 
significant effects on freedom from relapses compared to the $30 \mu \mathrm{g}$ group at all time points. There was a $32 \%$ relative reduction in the proportion of relapse-free patients treated with $44 \mu \mathrm{g}$ TIW at 24 weeks, and a $21 \%$ relative reduction at 48 weeks $(p=0.003)$. There was a $27 \%$ relative reduction in relapse rates at 24 weeks in the $44 \mu \mathrm{g}$ TIW treatment cohort $(\mathrm{p}=0.022)$; these relapse rates were 0.29 for the $44 \mu \mathrm{g}$ TIW group and 0.40 for the $30 \mu \mathrm{g}$ weekly group, however the reduction in relapses with $44 \mu \mathrm{g}$ TIW did not maintain significance at 48 weeks. At 24 weeks, the $44 \mu \mathrm{g}$ TIW group showed a significant effect on MRI lesions ( $p<0.0001)$. This effect was also maintained at 48 weeks $(\mathrm{p}<0.001)$. Injection site reactions, asymptomatic liver enzyme elevations, and altered leukocyte counts were more frequent in the $44 \mu \mathrm{g}$ TIW arm compared to the once weekly $30 \mu \mathrm{g}$ arm.

In 2005, an extension of the EVIDENCE trial was reported $^{59}$ in which patients in the $30 \mu \mathrm{g}$ IM weekly IFN $\beta$-1a group were permitted to cross over to $44 \mu \mathrm{g}$ SC TIW. The primary outcome measure was the annualized relapse rate compared to the rate prior to transition. Several secondary MRI endpoints were also included. The MRI evaluators remained blinded. Patients previously on $44 \mu \mathrm{g}$ TIW were allowed to remain on that dose. The post-transitional relapse rate decreased from 0.64 to 0.32 for patients switching to $44 \mu \mathrm{g}$ TIW ( $\mathrm{p}<0.001$ ) and from 0.46 to 0.34 for patients remaining on $44 \mu \mathrm{g}$ TIW $(\mathrm{p}=0.02)$. Patients converting to the higher dose regimen had fewer active T2 MRI lesions in comparison to pre-transition values $(\mathrm{p}=0.02)$. There were no significant MRI changes in patients maintained on high dose therapy. Patients switching to high dose therapy were noted to have an increase in mild to moderately severe adverse events.

A multicenter, randomized open label Danish trial ${ }^{60}$ recently compared IFN $\beta$-1a $\left(\right.$ Rebif $\left.^{\circledR}\right) 22 \mu$ g once weekly to the standard dose of IFN $\beta$-1b (Betaferon $^{\circledR}$ ) $250 \mu$ g every other day. Patients enrolled in this study had definite RRMS, EDSS scores of 0 to 5.5, and two relapses within the last two years. Blinding was not attempted in this trial except for MRI evaluations. Three hundred one patients were randomized to either dose regimen. A third cohort was included in this study, containing patients with newly diagnosed RRMS who refused randomization but were treated with $250 \mu \mathrm{g}$ IFN $\beta$-1b every other day. Annual relapse rates, time to first relapse, and time to sustained progression were virtually identical in the two arms of the randomized study. In the nonrandomized treatment group, the annual relapse rate was not significantly different, but there was a trend toward shorter time to progression. The conclusion of this study was that IFN $\beta-1 b$ $250 \mu$ g every other day was not clinically superior to IFN $\beta$-1a
$22 \mu \mathrm{g}$ once weekly, a surprising and uninterpretable finding in light of the INCOMIN and EVIDENCE trial results.

\section{Safety and tolerability}

The most common side effects of IFN $\beta$ therapy are flu-like symptoms, increased spasticity, hematologic and hepatic laboratory abnormalities, inflamed injection sites, cutaneous necrosis, and depression. ${ }^{61}$ Experience has shown that patient education about side effects prior to treatment, and proper management of adverse events, improves adherence to treatment. ${ }^{62,63}$ Injection site reactions are more common when beta interferon is administered SC compared to the IM route. These reactions are characterized by local pain, erythema, and induration. In the EVIDENCE trial, ${ }^{57} 83 \%$ of patients on SC IFN $\beta-1$ a versus $28 \%$ on IM IFN $\beta-1$ a experienced injection site reactions. Koch-Henriksen and colleagues ${ }^{60}$ reported the equal occurrence of injection site reactions in patients treated SC with either IFN $\beta-1$ a or IFN $\beta-1 b$. However, an observational prospective study of 306 Betaferon $^{\circledR}$ and 148 Rebif $^{\circledR}$ patients found significantly more pain-free days and fewer injection site reactions in the IFN $\beta$ - 1 b group. ${ }^{64}$

The cause of injection site reactions probably varies from one patient to another. Management strategies for treatment of these reactions include careful attention to aseptic technique, avoidance of intradermal injections, making sure the solution is completely dissolved and at room temperature, avoiding excessive sun exposure, local application of heat or cold, and use of nonsteroidal anti-inflammatory drugs (NSAIDS) to alleviate pain and prevent fever. Patients should be encouraged to keep a log of injection sites, making sure to alternate regularly to avoid skin breakdown. Injection site reactions usually diminish over a 3-6 month time period. Skin necrosis is a rare but serious complication of SC therapy, and to date no cases have been reported with the IM delivery route. Improper injection technique, including failure to alternate sites, has been shown to increase the risk of skin necrosis. If necrosis occurs, subcutaneous interferon should be discontinued and alternative therapy, including IM therapy, should be considered. Patients should be cautioned not to use topical steroids on these sites as they can delay recovery. ${ }^{61}$

Flu-like symptoms of chills, fatigue, headache, and myalgias can occur a few hours after administering IFN $\beta$, and may persist for 24 hours or more. Kümpfel and colleagues ${ }^{65}$ found IFN- $\beta$ injections at night led to increased IL-6 levels and temperature, correlating with decreased cortisol levels and more intense systemic side effects when compared to 
morning injections, with acute effects moderating after six months. This variation in time of injection administration may assist some patients with tolerability. Clinical experience has shown flu-like symptoms to become less frequent in most patients within three months of beginning therapy. Gradual dose escalation, as well as taking NSAIDS, reduces the occurrence of flu-like symptoms. ${ }^{66}$ Occasionally patients treated with IFN $\beta$ may notice an increase in spasticity during initiation of treatment. This typically occurs in patients who already have increased muscle tone and known temperature sensitivity, usually within hours of treatment, and lasting for days. Management with NSAIDS or antispasticity medications is often effective. Cases of hypothyroidism and hyperthyroidism are occasionally reported in patients on interferon therapy, and may require stopping therapy or switching to a non-IFN agent such as glatiramer acetate.

There is conflicting evidence to date regarding the relationship of IFN $\beta$ treatment to depression. Patients with known depression prior to treatment should be monitored carefully, and initiation of an antidepressant at the time of treatment should be considered. One should caution patients and family members to watch for depressive symptoms, and alert the prescribing provider should they occur. If patients on IFN $\beta$ therapy develop signs or symptoms of a mood disorder, the IFN $\beta$ should be discontinued, and if symptoms persist, treatment with psychotherapy, antidepressants, or both may be required. Occasionally, treatment may be re-instituted without recurrence of the depression.

Patients on IFN $\beta$ therapy commonly develop transient elevations of liver enzymes (AST and ALT), neutropenia, and less commonly, anemia. Tremlett and colleagues ${ }^{67}$ performed a retrospective chart review of $844 \mathrm{MS}$ patients treated with IFN $\beta-1 \mathrm{a}$ or IFN $\beta-1 \mathrm{~b}$ therapy, and found that $37 \%$ of patients developed new elevations of ALT. The effect of IM IFN was less than that of the SC IFNs on ALT levels. To date, two patients have been described with liver failure requiring transplantation, ${ }^{68}$ one of whom was concurrently taking nefazodone, another potentially hepatotoxic medication. ${ }^{69}$ No potential confounders could be identified in the second patient.

Francis and colleagues ${ }^{68}$ reviewed a pooled database of patients treated with IFN $\beta-1 \mathrm{a}$, and found ALT elevations in $59 \%$ of patients on IFN $\beta-1$ a $44 \mu \mathrm{g}$ TIW and $40 \%$ of patients taking IFN $\beta-1$ a $30 \mu \mathrm{g}$ per week. More than $50 \%$ of the elevations occurred within three months, $75 \%$ occurred within six months, and most elevations remitted spontaneously. Men were found to be more susceptible than women to liver enzyme elevations. Dosage reductions were necessary in $5 \%$ of treated patients, and universally led to normalization of values. Propionic acid derivatives taken concomitantly were the only medications shown to statistically be associated with increased risk of liver dysfunction. The authors recommended liver function analysis prior to treatment and at 1,3, and 6 months, with dose reductions for patients with ALT values of 5-20 times normal and discontinuation of treatment for those whose ALT levels are over 20 times normal. Despite these caveats, high dose IFN $\beta$ therapy is reasonably well tolerated, with an acceptable safety profile, as its continued long-term use in the MS population attests.

\section{Neutralizing antibodies}

The effect of neutralizing antibodies (NAbs) on IFN $\beta$ therapy remains a topic of controversy. In the IFN $\beta$-1a (Avonex ${ }^{\circledR}$ ) trial, ${ }^{37} 22 \%$ of patients developed NAbs; however an effect on endpoint measures could not be ascertained because of the short duration of the study. After reformulation, this product led to development of NAbs in only $5 \%-7 \%$ of patients. ${ }^{56,57}$ In the PRISMS-4 study, ${ }^{49}$ low dose interferon $22 \mu \mathrm{g}$ TIW was associated with the development of NAbs in $23.7 \%$ of patients while in the $44 \mu \mathrm{g}$ TIW group $14.3 \%$ developed NAbs. Though no clinical effect was noted at two years, a reduction in clinical outcome measures was found at four years. In the multicenter trial of IFN $\beta-1 b$ in RRMS, 38\% of patients developed NAbs by the third year with a noticeable attenuation of treatment effect. Perini and colleagues ${ }^{70}$ found $40 \%$ of $\beta-1 \mathrm{~b}$ patients, $6.7 \%$ of IM IFN $\beta-1 \mathrm{a}$, and $27 \%$ of SC IFN $\beta$-1a patients developed antibodies. This study found the presence of high titers of both binding and neutralizing antibodies to be indicative of loss of treatment effect. There are also data suggesting that $\mathrm{Nab}$ titers can revert to negative over time..$^{71}$ This was shown in the IFN $\beta-1 b$ follow-up study, ${ }^{72}$ in which $80 \%$ of NAb positive patients converted to antibody-negative status after 8 years despite continued therapy. NAbs to one IFN $\beta$ product cross-react with the others, ${ }^{73}$ so switching therapy to another IFN $\beta$ is not likely to be an effective strategy. In a recent phase III trial of a new and less immunogenic formulation of SC IFN $\beta$-1a $\left(\operatorname{Rebif}^{\circledR}\right)$, an interim analysis ${ }^{74}$ showed reduced antibody formation and improved tolerability. Publication of the results of this trial is awaited with interest.

The American Academy of Neurology recently reported the recommendations of a subcommittee focusing on the effect of NAbs on clinical and radiologic outcomes. ${ }^{75}$ The committee concurred that there is probably a reduction in efficacy of treatment because of NAbs. They also concurred 
that there is likely to be greater antibody production in response to IFN $\beta-1 b$ than to IFN $\beta-1 a$, and that IM IFN $\beta-1 a$ is clearly less immunogenic than other interferon therapies. Despite the consistent finding of NAb levels greater than 1:200 being associated with a reduction of efficacy, the committee was unable to make definite recommendations for changing therapy. This report highlights the fact that many questions remain unanswered in regard to the NAb issue.

The differences in assays used to measure NAbs, criteria for NAb positivity, and varying clinical assessment measures, make comparison between the studies difficult, if not impossible. Until clear data are available regarding the importance of neutralizing antibodies, decisions to change treatment should be based on the clinical picture. If a patient continues to have relapses despite IFN $\beta$ treatment, the physician should consider testing for NAbs and switching to another therapy. If NAbs are detected at a titer of 1:20 or greater on repeated testing, a non-interferon therapy such as glatiramer acetate should be considered. However, in a patient doing well clinically, there currently appears to be no benefit in testing for NAbs. The value of periodic MRI evaluation in the absence of clinical worsening has not been established.

\section{Quality of life}

Studies of IFN $\beta$ in the treatment of MS have shown benefit in relapse frequency and severity, as well as a reduction in hospitalizations. MS typically affects patients in the most productive years of their lives, and IFN $\beta$ therapy allows for fewer interruptions in activities of daily living. These studies have also shown excellent tolerability and relatively benign side effect profiles. The key to successful adherence to treatment is proper education about side effects and their management. ${ }^{62,63}$ Patients should be aware that beta interferon therapy is designated as pregnancy category $\mathrm{C}$, meaning that spontaneous abortions have been observed in IFN $\beta$-treated animals. A longitudinal, controlled cohort study found a decrease in mean birth weight and an increased risk of fetal loss in interferon exposed patients, even after correcting for potential cofounders. ${ }^{76}$ Patients are encouraged not to take any of the IFN $\beta$ products while breast feeding because the risks of transmission through breast milk are unknown.

A recent study showed that depression is the principal factor affecting quality of life in patients with $\mathrm{MS} .{ }^{77}$ The effect of interferon therapy on depression remains controversial. Symptoms such as suicidal ideation, decreased interest, feelings of guilt, loss of energy and concentration, and change in appetite may be signs of undiagnosed depression. Patients with these symptoms may require treatment with antidepressants, psychotherapy, or both and should be treated accordingly.

\section{Conclusions and recommendations}

The past decade has brought broader treatment options to physicians caring for patients with MS. Numerous randomized controlled trials of IFN $\beta$ have been published (Tables 1 and 2), confirming their efficacy on relapse rates, disease severity, and MRI outcomes. Unfortunately, long-term follow up assessments are few, and fraught with limitations. Those long-term studies that are available demonstrate that atrophy and disease progression continue despite early improvement in clinical and MRI outcomes. This suggests that other processes, in addition to inflammation, underlie the pathogenesis of MS, and indicate a need for additional treatment options. Until then, the current studies support treatment with IFN $\beta$ at the time of diagnosis of MS, as shown by the inability of placebo cohorts treated later to catch up to patients originally receiving interferon.

The relative importance of dose, frequency, and route of administration remains unclear. This is underlined by the recent study of double dose IFN $\beta-1 b$ and lack of statistical superiority found in patients treated with $500 \mu \mathrm{g}$ every other day compared to $250 \mu \mathrm{g}$ every other day, and to glatiramer acetate, on the risk of relapses. ${ }^{78}$ The relapse rate was very low in all study arms. Currently it appears that both dose and frequency of administration are important, and the effects of each cannot clearly be distinguished. Decisions about the choice of an IFN $\beta$ in each individual case should be made in the context of an informed discussion between physician and patient. Treatment should be customized to the patient with regard to frequency and route of administration, as well as side effects, in order to maximize compliance.

The decision to treat after a first demyelinating event (CIS), and before the diagnosis of MS is established, is also not obvious. The CHAMPS (35\% compared to 50\%), ETOMS (34\% compared to $45 \%$ ), and BENEFIT ( $15 \%$ compared to $31 \%$ ) studies all showed modest benefit at 2 years in preventing patients with CIS from having a second relapse. Those patients who have polysymptomatic disease and a larger T2 lesion load on MRI at the time of their first demyelinating event are more likely to convert to definite MS. Clinical suspicion and careful follow-up seems to be the best option in these cases, given that the alternative is prescribing a potentially unpleasant injectable medication that the patient may not need. There is no evidence to indicate that waiting a few months for the patient to meet the McDonald criteria for definite MS increases future risk compared to treating at the first sign of CIS. Furthermore, 
treatment at the CIS stage removes patients from the pool of potential RRMS subjects eligible for clinical trials. For these reasons, it is our practice to follow these patients with frequent MRIs and clinical examinations every 3-6 months.

The issue of NAbs also needs to be clarified. Any decision to change treatment remains a clinical one based on examination, history, and imaging. If a patient is doing well on treatment, knowing the NAb status is of little value. If a patient is doing poorly on treatment, it is time to change, regardless of NAbs.

Our understanding of MS with regard to pathophysiology and treatment is just beginning, and the prospects for further studies are exciting. The three available IFN $\beta$ preparations represent a major breakthrough in the history of MS therapy. In addition, by a process analagous to reverse engineering, they have given rise to a plethora of laboratory studies that have increased our understanding of MS pathogenesis and the mechanism of IFN action. However, numerous other compounds that may be more effective are currently in phase II and phase III clinical trials. Moreover, several of these are administered orally, an obvious advantage in terms of patient acceptance and compliance. Combination therapy also holds promise for MS, although well-designed and adequately controlled studies are difficult to conduct. Fortunately, placebo-controlled clinical trials are still considered ethical, at least under certain circumstances such as time-limited phase II studies and trials in SPMS and PPMS. ${ }^{79}$ Clinical trials of MS therapy continue to become increasingly complex, and a combination of placebo-controlled studies, trials comparing new agents to the best available current therapy, and combination or add-on studies will be necessary to provide the information needed to manage the broad spectrum of patients with this devastating disease

\section{Disclosure}

The authors report no conflicts of interest in this work.

\section{References}

1. Lublin FD, Reingold SC. Defining the clinical course of multiple sclerosis: results of an international survey. Neurology. 1996;46:907-911.

2. Fisniku LK, Brex PA, Altmann DR et al. Disability and T2 MRI lesions: a 20-year follow-up of patients with relapse onset multiple sclerosis. Brain. 2008;131:808-817.

3. Bar-Or A, Oliviera EML, Anderson DE, et al. Molecular pathogenesis of multiple sclerosis. J Neuroimmunol. 1999;100:252-259.

4. Conlon P, Oksenberg JR, Zhang J. The immunobiology of multiple sclerosis: an autoimmune disease of the central nervous system. Neurobiol Dis. 1999;6:149-166.

5. Martin R, Stürzebecher CS, McFarland HF. Immunotherapy of multiple sclerosis: Where are we? Where should we go? Nature Immunol. 2001;2:785-788

6. Yong VW, Chabot S, Stüve O, et al. Interferon beta in the treatment of multiple sclerosis: mechanisms of action. Neurology. 1998;51:692-689.
7. Noronha A, Toscas A, Jensen MA. Interferon $\beta$ decreases T-cell activation and interferon $\gamma$ production in multiple sclerosis. $J$ Neuroimmunol. 1993; 46:145-154.

8. Dhib-Jalbut S. Mechanisms of action of interferons and glatiramer acetate in multiple sclerosis. Neurology. 2002;58:S3-S9.

9. Graber JJ, Ford D, Zhan M, et al. Cytokine changes during interferonbeta therapy in multiple sclerosis: correlations with interferon dose and MRI response. J Neuroimmunol. 2007;185:168-174.

10. Stüve O, Dooley NP, Uhm JH, et al. Interferon $\beta$ - 1 b decreases the migration of T lymphocytes in vitro: effects on matrix metalloproteinase-9. Ann Neurol. 1996;40:853-863.

11. Leppert $\mathrm{D}$, Waubant $\mathrm{E}$, Bürk MR, et al. Interferon beta-1b inhibits gelatinase secretion and in vitro migration of human T cells: a possible mechanism for treatment efficacy in multiple sclerosis. Ann Neurol. 1996;40:846-852.

12. Stone LA, Frank JA, Albert PS, et al. The effect of interferon- $\beta$ on blood-brain barrier disruptions demonstrated by contrast-enhanced magnetic resonance imaging in relapsing-remitting multiple sclerosis. Ann Neurol. 1995;37:611-619.

13. Kraus J, Ling AK, Hamm S, et al. Interferon- $\beta$ stabilizes barrier characteristics for brain endothelial cells in vitro. Ann Neurol. 2004;56:192-205.

14. Calabresi PA, Tranquill LR, Dambrosia JM, et al. Increases in soluble VCAM-1 correlate with a decrease in MRI lesions in multiple sclerosis treated with interferon beta-1b. Ann Neurol. 1997;41: 669-674.

15. Graber J, Zhan M, Ford D, et al. Interferon $\beta$-1a induces increases in vascular cell adhesion molecule: implications for its mode of action in multiple sclerosis. J Neuroimmunol. 2005;161:169-176.

16. Yong VW. Differential mechanisms of action of interferon- $\beta$ and glatiramer acetate in MS. Neurology. 2002;59:802-808.

17. Sharief MK, Semra YK, Seidi OA, et al. Interferon-beta therapy down regulates the anti-apoptosis protein FLIP in T cells from patients with multiple sclerosis. J Neuroimmunol. 2001;121:102-110.

18. Hamamcioglu K, Reder AT. Interferon- $\beta$ regulates cytokines and BDNF: greater effect in relapsing than in progressive multiple sclerosis. Mult Scler. 2007;13:459-470.

19. Biernacki K, Antel AP, Blain M et al. Interferon beta promotes nerve growth factor secretion early in the course of multiple sclerosis. Arch Neurol. 2005;62:563-568.

20. Byskosh PV, Reder AT. Interferon- $\beta$ effects on cytokine mRNA in peripheral mononuclear cells in multiple sclerosis. Mult Scler. 1996;1:262-269.

21. Reder AT, Veichko S, Yanaguchi KD, et al. IFN $\beta-1 b$ induces transient and variable gene expression in relapsing-remitting multiple sclerosis patients independent of neutralizing antibodies or changes in IFN receptor RNA expression. J Interferon Cytokine Res. 2008;28:317-331.

22. Stürzebecher $\mathrm{S}$, Wandinger KP, Rosenwald A, et al. Expression profiling identifies responder and non-responder phenotypes to interferon- $\beta$ in multiple sclerosis. Brain. 2003;126:1419-1429.

23. Yamaguchi KD, Ruderman DL, Croze E, et al. IFN- $\beta$-regulated genes show abnormal expression in therapy-naïve relapsing-remitting MS mononuclear cells: gene expression analysis employing all reported protein-protein interactions. J Neuroimmunol. 2008;195:116-120.

24. Goodin DS, Frohman EM, Garmany GP, et al. Disease modifying therapies in multiple sclerosis. Report of the Therapeutics and Technology Assessment Subcommittee of the American Academy of Neurology and the MS council for Clinical Practice Guidelines. Neurology. 2002;58:169-178.

25. Knobler RL, Greenstein JL, Johnson KP, et al. Systemic recombinant human interferon- $\beta$ treatment of relapsing-remitting multiple sclerosis: Pilot study analysis and six-year follow up. J Interferon Res. 1993; 13:333-340.

26. IFNB Multiple Sclerosis Study Group. Interferon beta-1b is effective in relapsing remitting multiple sclerosis. I. Clinical results of a multicenter, randomized, double-blind, placebo-controlled trial. Neurology. 1993;43:662-667. 
27. Kurtzke JF. Rating neurologic impairment in multiple sclerosis: an expanded disability status scale (EDSS). Neurology. 1983;33: 1444-1452.

28. Paty DW, Li DKB, UBC MS/MRI Study Group, et al. Interferon beta-1b is effective in relapsing-remitting multiple sclerosis. II. MRI analysis results of a multi-center, randomized, double-blind, placebo-controlled trial. Neurology. 1993;43:662-667.

29. IFNB Multiple Sclerosis Study Group; University of British Columbia MS/MRI Analysis Group. Interferon beta- $1 \mathrm{~b}$ in the treatment of multiple sclerosis: Final outcome of the randomized controlled trial. Neurology. 1996;45:1277-1285.

30. European Study Group on Interferon $\beta-1 b$ in Secondary Progressive MS. Placebo-controlled multicenter randomized trial of interferon $\beta-1 \mathrm{~b}$ in treatment of secondary progressive multiple sclerosis. Lancet. 1998;352:1491-1497.

31. North American Study Group on Interferon beta-1b in Secondary Progressive MS. Interferon beta-1b in secondary progressive MS. Neurology. 2004;63:1788-1795.

32. Kappos L, Weinshenker B, Pozzilli C, et al. Interferon beta- $1 \mathrm{~b}$ in secondary progressive MS. A combined analysis of the two trials. Neurology. 2004;63:1779-1787.

33. Kappos L, Polman CH, Freedman MS, et al. Treatment with interferon beta 1-b delays conversion to clinically definite and McDonald MS in patients with clinically isolated syndromes. Neurology. 2006a;67:1242-1249.

34. Poser CM, Paty DW, Scheinberg L, et al. New diagnostic criteria for multiple sclerosis: guidelines for research protocols. Ann Neurol. $1983 ; 13: 227-231$.

35. McDonald WI, Compston A, Edan G, et al. Recommended diagnostic criteria for multiple sclerosis: guidelines from the International Panel on the diagnosis of multiple sclerosis. Ann Neurol. 2001;50: 121-127.

36. Kappos L, Freedman MS, Polman CH, et al. Effect of early versus delayed interferon beta- $1 \mathrm{~b}$ treatment on disability after a first clinical event suggestive of multiple sclerosis: a 3 year follow-up analysis of the BENEFIT study. Lancet. 2007;370:389-397.

37. Jacobs LD, Cookfair DL, Rudick RA, et al. Intramuscular interferon beta 1-a for disease progression in relapsing multiple sclerosis. The Multiple Sclerosis Collaborative Research Group (MSCRG). Ann Neurol. 1996;39:285-294.

38. Rudick RA, Goodkin DE, Jacobs LD, et al. Impact of interferon beta-1a on neurologic disability in relapsing multiple sclerosis. Neurology. 1997;49:358-363.

39. Fischer JS, Priore RL, Jacobs LD, et al. Neuropsychological effects of interferon $\beta-1 \mathrm{a}$ in relapsing multiple sclerosis. Ann Neurol. 2000;48:885-892.

40. Fisher E, Rudick RA, Simon JH, et al. Eight-year follow-up study of brain atrophy in patients with MS. Neurology. 2002;59: $1412-1420$.

41. Clanet M, Radue EW, Kappos L, et al. A randomized, double-blind, dose-comparison study of weekly interferon $\beta$-1a in relapsing MS. Neurology. 2002;59:1507-1517.

42. Clanet M, Kappos L, Hartung HP, et al. Interferon $\beta$-1a in relapsing multiple sclerosis: four-year extension of the European IFN $\beta$-1a dosecomparison study. Mult Scler. 2004;10:139-144.

43. Cohen JA, Cutter GR, Fischer JS, et al. Benefit of interferon $\beta-1 \mathrm{a}$ on MSFC progression in secondary progressive MS. Neurology. 2002;59:679-687.

44. Fischer JS, Rudick RA, Cutter GR, et al. The multiple sclerosis functional composite measure (MSFC): an integrated approach to MS clinical outcome assessment. Mult Scler. 1999;5:244-250.

45. Jacobs LD, Beck RW, Simon JH, et al. Intramuscular interferon beta-1a therapy initiated during a first demyelinating event in multiple sclerosis. N Engl J Med. 2000;343:898-904.

46. Leary SM, Miller DH, Stevenson VL, et al. Interferon $\beta$-1a in primary progressive MS. An exploratory, randomized, controlled trial. Neurology. 2003;60:44-51.
47. PRISMS (Prevention of Relapses and Disability by Interferon $\beta-1$ a Subcutaneously in Multiple Sclerosis) Study Group. Randomized double-blind placebo controlled study of interferon $\beta$-1a in relapsing/remitting multiple sclerosis. Lancet. 1998;352: $1498-1504$.

48. Li DKB, Paty DW, UBCMS/MRI Analysis Research Group and PRISMS Study Group. Magnetic resonance imaging results of the PRISMS trial: A randomized, double-blind, placebo-controlled study of interferonB1a in relapsing remitting multiple sclerosis. Ann Neurol. 1999;46: 197-206.

49. PRISMS Study Group; University of British Columbia MS/MRI Analysis Group. PRISMS-4: Long-term efficacy of interferon- $\beta$ - $1 \mathrm{a}$ in relapsing MS. Neurology. 2001;56:1628-1636.

50. Kappos L, Traboulsee A, Constantinescu C, et al. Long-term subcutaneous interferon beta-1a therapy in patients with relapsing remitting MS. Neurology. 2006b;67:944-953.

51. Once Weekly Interferon for MS Study Group (OWIMS). Evidence of interferon $\beta$-1a dose response in relapsing-remitting MS. Neurology. 1999;53:679-686.

52. Secondary Progressive Efficacy Clinical Trial of Recombinant 1-beta-1a in MS (SPECTRIMS) Study Group. Randomized controlled trial of interferon-beta-1a in secondary progressive MS. Clinical Results. Neurology. 2001;56:1496-1504.

53. Li DKB, Zhao GJ, Paty DW, et al. Randomized controlled trial of interferon-beta-1a in secondary progressive MS. Neurology. 2001;56:1505-1513.

54. Comi F, Fillipi M, Barkhof F, et al. Effect of early interferon treatment on conversion to definite multiple sclerosis: a randomized trial. Lancet. 2001;357:1576-1582.

55. Eillipi M, Rovaris $M$, Inglese $M$, et al. Interferon beta-1a for brain tissue loss in patients at presentation with syndromes suggestive of multiple sclerosis: a randomized, double-blind, placebo controlled trial. Lancet. 2004;364:1489-1496.

56. Durelli L, Verdun E, Barbero P, et al. Every other day interferon beta-1b vs once-weekly interferon beta-1a for multiple sclerosis: results of a 2-year prospective randomized multicenter study (INCOMIN). Lancet. 2002;359:1453-1460.

57. Panitch HS, Goodin DS, Francis G, et al. Randomized, comparative study of interferon $\beta 1$-a treatment regimens in MS: the EVIDENCE Trial. Neurology. 2002;59:1496-1506.

58. Panitch H, Goodin D, Francis G, et al. Benefits of high-dose, highfrequency interferon beta-1a in relapsing-remitting multiple sclerosis are sustained to 16 months: Final comparative results of the EVIDENCE trial. J Neurol Sci. 2005;239:67-74.

59. Schwid SR, Thorpe J, Sharief M, et al. Enhanced benefit of increasing interferon beta-1a dose and frequency in relapsing multiple sclerosis: the evidence study. Arch Neurol. 2005;62:785-792.

60. Koch-Henriksen N, S申rensen PS, Christensen T, et al. A randomized study of two interferon-beta treatments in relapsing-remitting multiple sclerosis. Neurology. 2006;66:1056-1060.

61. Walther EU, Hohlfeld R. Multiple sclerosis: side effects of interferon beta therapy and their management. Neurology. 1999;53: $1622-1627$.

62. Lublin FD, Whitaker JN, Eidelman BH, et al. Management of patients receiving interferon beta- $1 \mathrm{~b}$ for multiple sclerosis: report of a consensus conference. Neurology. 1996;46:12-18.

63. Munschauer FE, Kinkel RP. Managing side effects of interferon-beta in patients with relapsing-remitting multiple sclerosis. Clin Ther. 1997; 19:883-893.

64. Baum K, O'Leary C, Ferrer FC, et al. Comparison of injection site pain and injection site reactions in relapsing-remitting multiple sclerosis patients treated with interferon beta-1a or 1b. Mult Scler. 2007;13:1153-1160.

65. Kümpfel T, Schwan M, Pollmächer T, et al. Time of interferon- $\beta$-1a injection and duration of treatment affect clinical side effects and acute changes of plasma hormone and cytokine levels in multiple sclerosis patients. Mult Scler. 2007;13:1138-1145. 
66. Rice GP, Ebers GC, Lublin FD, et al. Ibuprofen treatment versus gradual introduction of interferon $\beta 1$-b in patients with MS. Neurology. 1999;52:1893-1895.

67. Tremlett HL, Yoshida EM, Oger J. Liver injury associated with the $\beta$-interferons for MS: a comparison between three products. Neurology. 2004;62:628-631.

68. Francis GS, Grumser Y, Alteri E, et al. Hepatic reactions during treatment of multiple sclerosis with interferon- $\beta$-1a: incidence and clinical significance. Drug Safety. 2003;26:815-827.

69. Yoshida EM, Rasmussen SL, Steinbrecher UP, et al. Fulminant liver failure during interferon-beta treatment of multiple sclerosis. Neurology. 2001;56:1416.

70. Perini P, Calabrese M, Biasi G, et al. The clinical impact of interferon beta antibodies in relapsing-remitting MS. J Neurol. 2004;251:305-309.

71. Rice GP, Paszner B, Oger J, et al. The evolution of neutralizing antibodies in multiple sclerosis patients treated with interferon $\beta-1 \mathrm{~b}$. Neurology. 1999;52:1277-1279.

72. Rice G. The significance of neutralizing antibodies in patients with multiple sclerosis treated with interferon beta. Arch Neurol. 2001;58:1297-1298

73. Khan OA, Dhib-Jalbut SS. Neutralizing antibodies to interferon beta-1a and interferon beta-1b in MS patients are cross-reactive. Neurology. 1998;51:1698-1702.
74. Giovannoni G, Barbarash OL, Jaber A, et al. Reduced immunogenicity with a new formulation of interferon of interferon-beta-1a $\left(\right.$ Rebif $\left.^{\circledR}\right)$ : 24-week results of a phase IIIb study. Mult Scler. 2006; 12(Suppl 1):S192.

75. Goodin DS, Frohman EM, Hurwitz B, et al. Neutralizing antibodies to interferon beta: assessment of their clinical and radiographic impact: an evidence report. Report of the Therapeutics and Technology Assessment Subcommittee of the American Academy of Neurology. Neurology. 2007;68:977-984.

76. Boskovic R, Wide R, Wolpin J, et al. The reproductive effects of beta interferon therapy in pregnancy: a longitudinal cohort. Neurology. 2005;65:807-811.

77. Patti F, Russo P, Pappalardo A, et al. Predictors of quality of life among patients with multiple sclerosis: an Italian cross-sectional study. J Neurol Sci. 2007;252:121-129.

78. Bayer Schering Pharma AG. BEYOND study: results do not support regulatory filing for Betaferon ${ }^{\circledR} 500 \mu \mathrm{g}$. Press Release. Leverkusen, Germany: Bayer Schering Pharma AG; 2007.

79. Lublin FD, Reingold SC, National Multiple Sclerosis Society (USA) Task Force on Placebo-Controlled Clinical Trials in MS. Placebocontrolled clinical trials in multiple sclerosis: ethical considerations. Ann Neurol. 2001;49:677-681.
Biologics: Targets \& Therapy

\section{Publish your work in this journal}

Biologics: Targets \& Therapy is an international, peer-reviewed journal focusing on the patho-physiological rationale for and clinical application of Biologic agents in the management of autoimmune diseases, cancers or other pathologies where a molecular target can be identified. This journal is indexed on PubMed Central, CAS,

\section{Dovepress}

EMBase, Scopus and the Elsevier Bibliographic databases. The manuscript management system is completely online and includes a very quick and fair peer-review system, which is all easy to use. Visit http://www.dovepress.com/testimonials.php to read real quotes from published authors. 